\title{
A LIGA INTERDISCIPLINAR EM SAÚDE MENTAL E SUAS CONTRIBUIÇÕES EM TEMPOS DE COVID-19
}

\section{LA LIGA INTERDISCIPLINARIA EN SALUD MENTAL Y SUS CONTRIBUCIONES EN TIEMPOS DE COVID-19}

\author{
THE MENTAL HEALTH INTERDISCIPLINARY LEAGUE AND ITS \\ CONTRIBUTIONS IN TIMES OF COVID-19
}

\author{
Eliany Nazaré OLIVEIRA ${ }^{1}$ \\ Carla Suyane Gomes de ANDRADE ${ }^{2}$ \\ Letícia Mara Cavalcante LIMA ${ }^{3}$
}

RESUMO: A Organização Mundial da Saúde (OMS) declarou, em janeiro de 2020, que o surto da doença causada pelo novo coronavírus constituiu uma Emergência de Saúde Pública de Importância Internacional, o mais alto nível de alerta da Organização, conforme previsto no Regulamento Sanitário Internacional. A saúde mental da população durante uma pandemia de grande magnitude como esta implica em uma perturbação psicossocial que pode ultrapassar a capacidade de enfrentamento da população afetada. Este artigo possui como objetivo relatar a experiência da Liga Interdisciplinar em Saúde Mental (LISAM) da Universidade Estadual Vale do Acaraú, durante a pandemia do novo coronavírus. A Universidade, ao desenvolver ações de extensão durante a pandemia, compromete-se em sustentar seu valor social e mostrar-se atenta para o delicado momento histórico mundial. $\mathrm{O}$ relato está constituído de três sessões: criação, função e perspectivas; contribuições da liga durante a pandemia e pesquisar é preciso. Ao desenvolver atividades de extensão durante a pandemia, oportunizou aos seus integrantes um espaço fértil de aprendizagem no âmbito do ensino, pesquisa e extensão, contribuindo para a formação de profissionais com senso de responsabilidade social e compromisso com a cidadania.

PALAVRAS-CHAVE: Saúde Mental. Extensão Comunitária. Educação Superior. COVID19. Pandemia.

RESUMEN: La Organización Mundial de la Salud (OMS) declaró, en enero de 2020, que el brote de la enfermedad causada por el COVID-19 constituye una Emergencia de Salud Pública de Importancia Internacional, el nivel más alto de alerta de la Organización, conforme lo previsto en el Reglamento Sanitario Internacional. La salud mental de la población durante una pandemia de gran magnitud como esta conlleva una perturbación psicosocial que puede ultrapasar la capacidad de enfrentamiento de la población afectada.

${ }^{1}$ Universidade Estadual Vale do Acaraú (UVA), Sobral - CE - Brasil. Docente no Curso de Enfermagem. Doutora em Enfermagem (UFC). ORCID: http://orcid.org/0000-0002-6408-7243. E-mail: elianyy@gmail.com

${ }^{2}$ Universidade Estadual Vale do Acaraú (UVA), Sobral - CE - Brasil. Graduanda no Curso de Enfermagem. Integrante da Liga Interdisciplinar em Saúde Mental. ORCID: https://orcid.org/0000-0003-2492-338X. E-mail: carlasuyane@hotmail.com

${ }^{3}$ Universidade Estadual Vale do Acaraú (UVA), Sobral - CE - Brasil. Graduanda no Curso de Enfermagem. Integrante da Liga Interdisciplinar em Saúde Mental. ORCID: https://orcid.org/0000-0002-7258-5787. E-mail: leticiamara55@gmail.com 
Este artículo tiene como objetivo relatar la experiencia de la Liga Interdisciplinaria en Salud Mental (LISAM) de la Universidad Estadual Vale de Acaraú, durante la pandemia del nuevo coronavirus. La Universidad al desarrollar acciones de extensión durante la pandemia, se compromete a sustentar su valor social y muestra su preocupación con el delicado momento histórico mundial. El relato está constituido por tres sesiones: creación, función y perspectivas; contribuciones de la liga durante la pandemia e investigar es necesario. Al desarrollar actividades de extensión durante la pandemia, proporcionó a sus integrantes un espacio fértil de aprendizaje en el ámbito de la enseñanza, investigación y extensión, contribuyendo para la formación de profesionales com sentido de responsabilidad social y compromiso con la ciudadanía.

PALABRAS CLAVE: Salud mental. Extensión comunitaria. Educación superior. COVID-19. Pandemia

ABSTRACT: The World Health Organization (WHO) has declared in January 2020 that the outbreak caused by Coronavirus infections constituted a Public Health Emergency of International Concern, the highest warning level of the Organization, as predicted in the International Sanitary Regulation. The mental health of a population, during a great magnitude pandemic such as this, implies in a psychosocial disorder which can exceed the confrontation capacity of the affected population. This article aims to report the experience of the Mental Health Interdisciplinary League (MHIL) from Vale do Acaraú State University, during the new coronavirus pandemic. By promoting extension actions during the pandemic and by committing itself to sustain its social value, the university shows itself to be attentive to this delicate world-historical moment. This report is constituted in three sections: creation, function, and perspectives; the league's contribution during the pandemic; and researching is necessary. By developing extension activities during the pandemic, it provided its members with a fertile space for learning in the field of teaching, research and extension, contributing to the formation of professionals with a sense of social responsibility and commitment to citizenship.

KEYWORDS: Mental health. Community-institutional Relations. Higher education. COVID19. Pandemic.

\section{Introdução}

O mundo mudou a partir de dezembro de 2019. A pandemia do novo coronavírus tem acionado instituições internacionais, governos, profissionais e populações para instituírem estratégias de contenção, uma vez que os deslocamentos aceleram ou retardam o crescimento dos casos de infecções (DARSIE; WEBER, 2020). Estamos atravessando a maior crise sanitária nunca vivenciada até então. Em janeiro de 2020, a Organização Mundial da Saúde (OMS) oficializou a existência da doença causada pelo COVID-19 e declarou o estado de Emergência em Saúde Pública de Importância Internacional (ORGANIZAÇÃO MUNDIAL DA SAÚDE, 2020; ORGANIZAÇÃO PAN-AMERICANA DE SAÚDE, 2020). 
Estudos já demonstram importantes impactos na saúde mental da população mundial. De acordo com Schmidt et al. (2020), a rápida disseminação do novo coronavírus por todo o mundo, as incertezas sobre como controlar a doença e sobre sua gravidade, além da imprevisibilidade acerca do tempo de duração da pandemia e dos seus desdobramentos caracterizam-se como fatores de risco à saúde mental da população geral. Já Oliveira (2020) enfatiza a possibilidade de sofrimento psíquico e surgimentos de transtornos mentais. Nesse sentido, muito necessita ser feito. Além das frentes emergenciais de trabalho durante e póspandemia.

Quais as contribuições da universidade em uma situação de crise sanitária no cenário brasileiro? É exatamente neste momento que a Liga Interdisciplinar em Saúde Mental da Universidade Estadual Vale do Acaraú (UVA) se reorganiza e traz as suas contribuições para o enfrentamento das situações adversas provocadas pela pandemia do novo coronavírus. Assim, a extensão universitária apresenta-se como um dos caminhos viáveis para dar suporte e apoio às necessidades advindas da sociedade (HUNGER et al., 2014).

Para Oliveira et al. (2020), esta crise sanitária irá contribuir para a busca de novos desafios e aprendizados, possibilitando a reflexão quanto às fragilidades até então não reconhecidas. E será exatamente esta a oportunidade que as universidades terão para demonstrar sua função na pesquisa, extensão e ensino.

Para Araújo et al. (2019), as Ligas Acadêmicas caracterizam-se por suas potencialidades para a transformação do cenário de práticas de saúde em vários cenários e pontos da rede de atenção à saúde. Criam possibilidades reais para a implementação do ensino, pesquisa, extensão na universidade e consolidam a interlocução com a sociedade.

No contexto da extensão universitária, incluem-se as Ligas Acadêmicas (LA) que possuem como objetivo aproximar o estudante da prática de atenção à saúde, propiciar a indissociabilidade do tripé da formação (ensino, pesquisa, extensão), oferecer diversidade de cenários de práticas, formar para a saúde, aprender a fazer e aprender a cuidar do outro (SILVA; FLORES, 2015).

O Plano Nacional de Extensão Universitária, lançado pelo Ministério da Educação, reconhece a extensão como um processo educativo que possibilita a relação transformadora entre universidade e sociedade, articulando-se com o ensino e a pesquisa. $\mathrm{O}$ documento é fortalecido pela Resolução $\mathrm{n}^{\circ}$ 7, de 18 de dezembro de 2018 que Estabelece as Diretrizes para a Extensão na Educação Superior Brasileira e regimenta o disposto na Meta 12.7 da Lei $n^{\circ}$ 13.005/2014 a qual aprova o Plano Nacional de Educação PNE 2014 - 2024 (BRASIL, 2018). 
As instituições devem garantir sua função junto à sociedade que deve estar atenta a essa nova conjectura social que se formou durante esse tempo de pandemia. A extensão universitária poderá ser o caminho para a promoção do desenvolvimento humano e ambiental dentro dessa nova realidade (SILVA, 2020).

Vale ressaltar que a Extensão Universitária deve considerar um cenário excepcional e emergencial no qual temos vivido em virtude da pandemia do COVID-19. O momento exige vigilância, cuidados sanitários e medidas profiláticas. $\mathrm{O}$ isolamento social restringiu o nosso ir e vir, ou seja, foi instituída uma barreira sobre o livre deslocamento e interação social. Este novo cenário tem alterado profundamente a forma como iremos atuar na sociedade. $\mathrm{O}$ fenômeno pandêmico também evoca a consequente criação de novos paradigmas de ação coletiva e que tocam diretamente as atividades extensionistas (SERRÃO, 2020).

Alguns autores consideram a extensão universitária como uma inovação pedagógica por estabelecer uma relação dialógica entre a universidade e a sociedade, por meio do protagonismo estudantil, da reconstrução dos saberes, da descolonização dos currículos, das práticas criativas da docência e da democratização epistemológica das instituições de ensino superior (SILVA; CAMPANI; NEGREIROS, 2020).

Com esta perspectiva, este artigo possui o seguinte objetivo: relatar a experiência da Liga Interdisciplinar em Saúde Mental durante a pandemia do novo coronavírus.

\section{Criação, função e perspectivas}

A Liga Interdisciplinar em Saúde Mental (LISAM), vinculada à Universidade Estadual Vale do Acaraú (UVA), iniciou suas atividades em 2017. O processo de Implantação e desenvolvimento da LISAM contou com o protagonismo dos estudantes do Curso de Enfermagem desta instituição, com mobilização para construção de todo o processo inicial previsto nos trâmites institucionais, como: construção coletiva do estatuto e aprovação deste junto à Pró-reitoria de Extensão e Cultura (PROEX) da UVA, assim como elaboração e execução do primeiro edital de seleção (OLIVEIRA et al., 2019).

A LISAM em seu primeiro ano de criação agregou apenas estudantes da UVA e dos Cursos de Enfermagem, Pedagogia e Educação Física. Diante de sua essência interdisciplinar, percebe-se a necessidade da participação de outros cursos. Assim, no segundo ano, fica mais potente com a inclusão da Universidade Federal do Ceará (UFC) e passa a ser constituída por estudantes dos seguintes cursos: enfermagem, educação física, psicologia, direito e pedagogia, o que fortaleceu a característica interdisciplinar (OLIVEIRA et al., 2019). 
Nessa perspectiva, compreende-se que a interdisciplinaridade envolve um novo modo de pensar a realidade, que resulta em troca, reciprocidade e integração entre diferentes áreas de conhecimento, assim como o ensejo pela produção de novos conhecimentos e a resolução de problemas, de modo global e abrangente (SANTOS; VIEIRA, 2011).

A LISAM apresenta atualmente a seguinte estruturação: um professor coordenador orientador, um presidente, um vice-presidente, um diretor financeiro, um diretor de ensino, pesquisa e extensão, um diretor administrativo, um diretor de informática e marketing, cinco profissionais preceptores e dez estudantes/ligantes. Este formato constituído está previsto no estatuto, além dos princípios que norteiam as ações, os requisitos de admissão e exclusão dos membros, os direitos e deveres, o funcionamento da Liga, as condições para disposições regimentais e dissolução e a forma de gestão administrativa. Com esta perspectiva, tenta desenvolver sua missão tendo como meta o princípio da indissociabilidade entre ensino, pesquisa e extensão; assumem um caráter extracurricular e complementar; e suas ações são de natureza teórica e prática. As atividades são desenvolvidas buscando-se a articulação entre o ensino, pesquisa e extensão (CASTRO; ALVES, 2017).

Assim, a interdisciplinaridade, como princípio das atividades extensionistas na área da saúde, intenta promover a mobilização e a articulação de todas as condições materiais e humanas necessárias para garantirem o avanço dos processos de ensino, orientada para uma formação mais efetiva do estudante, de modo a contribuir para tornar todos eles capazes de enfrentar, adequadamente, os desafios impostos pelo mercado de trabalho e necessidades da sociedade globalizada (SANTOS; VIEIRA, 2011).

A LISAM assumiu em seu estatuto o compromisso de desenvolver atividades para o fortalecimento das estratégias promotoras de saúde mental, tendo em vista as vulnerabilidades locais; propõe a construção de práticas interdisciplinares, orientadas pelo princípio da universalidade e equidade e visa envolver os estudantes em atividades no âmbito da Saúde Mental da população. A principal missão é promoção da saúde mental comunitária (OLIVEIRA et al., 2019).

\section{Contribuições da liga durante a pandemia}

O fenômeno da pandemia do coronavírus tem atingido todo o tecido social, incluindo praticamente todas as áreas da vida coletiva ou individual, com repercussões na esfera da saúde mental. Nas crises sanitárias, o número de indivíduos psicologicamente afetados pode ser maior que o de pessoas acometidas pela infecção, sendo estimado que de um terço à 
metade da população possa apresentar consequências psicológicas e psiquiátricas caso não receba cuidados adequados (LIMA, 2020).

Compreende-se que a pandemia do novo coronavírus poderá contribuir para o aperfeiçoamento da prática em saúde mental e da pesquisa em situações de crise, emergência e grandes desastres (OLIVEIRA, 2020).

De acordo com Silva (2020), a extensão universitária pode e deve promover o desenvolvimento humano nesta nova realidade, pois as oportunidades suplantam os desafios. Os desafios anteriores eram de natureza computacionais-tecnológicos, mas agora estamos na era das informações em tempo real, as tecnologias já estão desenvolvidas e acessíveis e os recursos computacionais estão em nossas mãos.

Quadro 1 - Apresentação dos vídeos construídos e publicados nas redes sociais durante a pandemia do novo coronavírus

\begin{tabular}{|c|l|}
\hline TEMÁTICA & \multicolumn{1}{|c|}{ OBJETIVO } \\
\hline Autocuidado & $\begin{array}{l}\text { Incentivar alguns cuidados que as pessoas podem ter } \\
\text { consigo mesmas, mostrando a importância do } \\
\text { autocuidado, tendo em vista o momento difícil que } \\
\text { estamos vivenciando. }\end{array}$ \\
\hline Alimentação e saúde mental & $\begin{array}{l}\text { Estimular o hábito da alimentação saudável, } \\
\text { destacando alguns alimentos que comprovadamente } \\
\text { beneficiam a saúde física e mental. }\end{array}$ \\
\hline Estresse e ansiedade & $\begin{array}{l}\text { Informar sobre as características do estresse e } \\
\text { ansiedade e seus efeitos nocivos no organismo. E } \\
\text { alertar sobre seu surgimento no período de isolamento } \\
\text { social e quarentena. }\end{array}$ \\
\hline Autoestima & $\begin{array}{l}\text { Estimular a análise crítica da natureza da autoestima, } \\
\text { no individual, por meio da apresentação da definição } \\
\text { do termo, da sua relevância para a saúde mental. }\end{array}$ \\
\hline Atividade física e saúde mental & $\begin{array}{l}\text { Destacar a relevância da atividade física para saúde } \\
\text { fisica e mental, especialmente durante isolamento } \\
\text { social e quarentena. }\end{array}$ \\
\hline Autoconhecimento & $\begin{array}{l}\text { Instigar a busca do autoconhecimento, por meio da } \\
\text { promoção da compreensão do termo e de sua } \\
\text { importância para a saúde mental. }\end{array}$ \\
\hline
\end{tabular}

Fonte: Elaborado pelas autoras

As temáticas dos vídeos foram escolhidas com base nas principais necessidades da população com a chegada abrupta da crise sanitária trazida pelo novo coronavírus. Durante uma pandemia, é esperado que estejamos frequentemente em estado de alerta, preocupados, confusos, estressados e com sensação de falta de controle frente às incertezas do momento. Existe uma estimativa que entre um terço e metade da população exposta a uma epidemia pode vir a sofrer alguma manifestação psicopatológica, principalmente se não for feita nenhuma intervenção de cuidados psicossociais. Os fatores que influenciam o impacto 
psicossocial estão relacionados à magnitude da epidemia e ao grau de vulnerabilidade em que a pessoa se encontra no momento (FIOCRUZ, 2020a).

Os temas dos vídeos produzidos e publicados nas redes sociais possuíam o objetivo de amenizar alguns efeitos negativos provocados pelo isolamento social e quarentena. De acordo com a Fiocruz (2020a), algumas reações comportamentais mais presentes neste momento são: alterações ou distúrbios de apetite (falta de apetite ou apetite em excesso); alterações ou distúrbios do sono (insônia, dificuldade para dormir ou sono em excesso, pesadelos recorrentes); conflitos interpessoais (com familiares, equipes de trabalho...) e pensamentos recorrentes relacionados à morte e ao morrer.

Quadro 2 - Apresentação do ciclo teórico desenvolvido pela LISAM durante a pandemia do novo coronavírus

\begin{tabular}{|c|c|}
\hline TEMÁTICA & OBJETIVO \\
\hline $\begin{array}{c}\text { O consumo de álcool e outras drogas durante o } \\
\text { isolamento social }\end{array}$ & $\begin{array}{l}\text { Discutir em que medida o isolamento social e } \\
\text { quarentena podem influenciar no aumento ou redução } \\
\text { de substâncias psicoativas. }\end{array}$ \\
\hline Violência doméstica durante o isolamento social & $\begin{array}{l}\text { Debater a saúde mental dos indivíduos, diante do } \\
\text { momento de isolamento social, além disso refletir } \\
\text { sobre as mudanças, adaptações e consequências na } \\
\text { saúde física e mental da população. }\end{array}$ \\
\hline Saúde mental em tempos de pandemia & $\begin{array}{l}\text { Apresentar características } \text { epidemiológicas } \\
\text { psicossociais e suas interfaces com a pandemia e a } \\
\text { saúde mental }\end{array}$ \\
\hline $\begin{array}{l}\text { "O mal-estar na civilização em Freud" discutindo } \\
\text { saúde mental nos dias atuais. }\end{array}$ & $\begin{array}{l}\text { Dialogar com a obra do psicanalista Sigmund Freud, } \\
\text { "o mal-estar na civilização", com o contexto atual } \\
\text { vivido. Com intuito de refletir e compreender a } \\
\text { presença dos sentimentos negativos. }\end{array}$ \\
\hline $\begin{array}{l}\text { Política nacional sobre drogas: características em } \\
\text { tempos de pandemia do novo coronavírus }\end{array}$ & $\begin{array}{l}\text { Conversar sobre a política nacional de redução de } \\
\text { danos e suas características e ressignificações durante } \\
\text { o isolamento social }\end{array}$ \\
\hline Ansiedade em temos de pandemia & $\begin{array}{l}\text { Refletir sobre os principais motivos que podem gerar } \\
\text { ansiedade durante a pandemia. Esclarecer os sinais e } \\
\text { sintomas e informar as principais medidas de proteção }\end{array}$ \\
\hline
\end{tabular}

Fonte: Elaborado pelas autoras

Com o início da pandemia, as atividades do eixo ensino tiveram que ser reorganizadas, tendo em vista a necessidade de novos conhecimentos, habilidades e competências. Nesta perspectiva, para Cavalcante et al. (2018), as principais contribuições das ligas para a formação em saúde são a promoção de uma formação em sintonia com a realidade em que estes profissionais atuarão. Então, a necessidade de conhecimentos envolvendo o contexto pandêmico foi instituída para acompanhar as demandas psicossociais e epidemiologias trazidas pela pandemia. Deste modo, contribui-se para a formação técnico-científica e 
humanística dos integrantes, tendo como meta a promoção da saúde mental da população (SILVA et al., 2020).

A crise sanitária e humanitária provocada pela COVID-19 atinge de forma significativa um grande número de pessoas e impõe novas regras e comportamentos sociais até então não experimentados (DUARTE et al., 2020). Novos conhecimentos devem ser produzidos para o enfretamento das demandas atuais.

Ligas Acadêmicas são potencialmente benéficas para os estudantes em formação e para a sociedade em geral, pois são fonte de conhecimentos e consequentemente de ações em prol dos indivíduos, famílias e comunidades (QUEIROZ et al., 2014).

Quadro 3 - Apresentação das postagens nas redes sociais durante a pandemia do novo coronavírus

\begin{tabular}{|l|l|}
\hline \multicolumn{1}{|c|}{ TEMÁTICAS PUBLICADAS NO INSTAGRAM } & \multicolumn{1}{|c|}{ OBJETIVO } \\
\hline $\begin{array}{l}\text { Indicações de artigos relevantes à saúde mental em } \\
\text { meio ao isolamento social. }\end{array}$ & $\begin{array}{l}\text { Repassar conhecimento sobre o isolamento social e } \\
\text { estratégias do cuidado psíquico em situação de } \\
\text { pandemia com a divulgação de artigos pertinentes. }\end{array}$ \\
\hline $\begin{array}{l}\text { Texto relevante sobre a saúde mental durante a } \\
\text { pandemia }\end{array}$ & $\begin{array}{l}\text { Tratar de dicas de como diminuir a ansiedade. Tendo } \\
\text { o auxílio de páginas no Instagram que dispõem de } \\
\text { técnicas que diminuem a ansiedade. }\end{array}$ \\
\hline Texto sobre a procrastinação & $\begin{array}{l}\text { Esclarecer a definição da procrastinação, como ela } \\
\text { pode se apresentar no cotidiano e dicas para contê-la. }\end{array}$ \\
\hline Texto sobre a autocobrança excessiva & $\begin{array}{l}\text { Descrever como a autocobrança excessiva pode se } \\
\text { apresentar na rotina e dicas de como refreá-la. }\end{array}$ \\
\hline Artigo relevante ao isolamento social & $\begin{array}{l}\text { Indicar artigos relevantes sobre o isolamento social e } \\
\text { exibir um fluxonagrama de suporte social e } \\
\text { psicológico de atendimento tanto para as pessoas em } \\
\text { crise, como para os profissionais de saúde. }\end{array}$ \\
\hline Dicas de canais de apoio psicológico & $\begin{array}{l}\text { Informar sobre os diversos canais de apoio } \\
\text { psicológico que se pode ter em meio ao isolamento } \\
\text { social, via internet e ao telefone, com escuta } \\
\text { qualificada. }\end{array}$ \\
\hline Texto sobre o estigma social & $\begin{array}{l}\text { Abordar sobre o estigma social, sua definição, como } \\
\text { pode se apresentar e dicas para evitá-lo. }\end{array}$ \\
\hline
\end{tabular}

Fonte: Elaborado pelas autoras

As postagens de assuntos relevantes nas redes sociais é uma das estratégias de trabalho da LISAM para o enfrentamento das repercussões da pandemia na vida das pessoas. Neste sentido, as atividades no âmbito da saúde mental encontram-se alinhadas com o eixo central proposto no estatuto da liga: promoção da saúde mental comunitária.

Redes sociais são plataformas com alta velocidade na geração de dados, com postagens feitas a todo instante. Com a pandemia e milhares de pessoas em diversas formas de isolamento, existe um maior uso de redes sociais. Com a popularização do acesso à 
internet, as redes sociais estão entre as principais formas de comunicação atuais (STATISTA, 2020).

As redes sociais foram o principal local mobilização da LISAM. No cenário instituído pela pandemia, em que o uso generalizado de tecnologia da informação pode ser destacado como uma abordagem fundamental para lidar com a crise, o uso das mídias sociais pode aproximar as pessoas e tornar a comunicação mais efetiva (SHIOZAWA; UCHIDA, 2020).

Alguns estudos estão evidenciando quadros com os seguintes sinais e sintomas: estresse, medo, pânico, ansiedade, culpa e tristeza que geram sofrimento psíquico. Em determinados indivíduos pode ocasionar o surgimento de transtornos de pânico e transtornos de ansiedade (PEREIRA et al., 2020; SOUADKA et al., 2020).

Existe uma grande sobrecarga psicossocial provocada pela COVID-19. As medidas de controle e prevenção da infecção são responsáveis por vários comportamentos e atitudes. Os esforços para responder a essas necessidades de saúde mental garantem aos pesquisadores uma importante oportunidade para desenvolver o que sabemos e para alcançar os objetivos da saúde mental mundial (KOLA, 2020).

\section{Pesquisar é preciso}

As atividades são desenvolvidas buscando a articulação entre o ensino, pesquisa e extensão, levando em consideração a indissociabilidade entre eles. O princípio constitucional dessa indissociabilidade é um processo interdisciplinar educativo, cultural, científico e político que promove a interação transformadora entre universidade e outros setores da sociedade (BEGO; SILVA, 2018).

A situação sanitária e epidemiológica desencadeada pela pandemia do novo coronavírus impulsionou a iniciativa que culminou no desenvolvimento do estudo intitulado: Repercussões da pandemia do novo coronavírus na saúde mental de estudantes do ensino superior. Com o seguinte objetivo principal: analisar situação de saúde mental de estudantes do ensino superior do estado do Ceará durante a pandemia do novo coronavírus.

A pesquisa apresenta as questões norteadoras: como está a saúde mental dos estudantes universitários que tiveram suas rotinas drasticamente modificadas? Quais os principais problemas enfrentados por estes estudantes durante o isolamento social? Em que medida a pandemia e a necessidade de isolamento social está impactando na sua saúde mental? 
Utiliza-se um questionário do perfil sociodemográfico e situacional em relação à pandemia e isolamento social. E o instrumento principal é o Mental Health Inventory (ISM), desenvolvido para a investigação epidemiológica por vários investigadores no âmbito do Health Insurance Study da Rand Corporation. Ele tem como escopo avaliar a saúde mental na população em geral, ou em grupos populacionais específicos, numa perspectiva bidimensional, incluindo aspectos positivos e negativos (RIBEIRO, 2011).

O ISM se mostra relevante para avaliar saúde mental a partir das experiências de saúde da pessoa, diferenciando níveis de funcionamento positivo ou negativo, sendo esta capacidade discriminante essencial para distinguir pessoas com necessidades de ajuda. $\mathrm{O}$ instrumento contém 38 itens distribuídos em cinco escalas, que se agrupam em duas grandes dimensões: Distress Psicológico (negativa) que inclui indicadores negativos tradicionais do sofrimento psicológico ou estados emocionais e mentais negativos. Contém as escalas de Ansiedade (10 itens), Depressão (5 itens), Perda de Controle Emocional e Comportamental (9 itens); BemEstar Psicológico (positiva), que contém a tônica contemporânea na saúde mental positiva ou estados positivos que incluem as escalas Afeto Geral Positivo (11 itens) e Laços Emocionais (3 itens)

O ISM é avaliado por meio de escala ordinal do tipo Likert, que varia entre cinco e seis possibilidades. O resultado de cada dimensão positiva e negativa deriva do somatório bruto dos itens que lhe correspondem. O somatório das duas dimensões fornece o Índice de Saúde Mental. Os valores mais elevados correspondem a melhores níveis de saúde mental (RIBEIRO, 2011).

Diante do cenário atípico, com a obrigatoriedade do isolamento social e o encerramento das aulas presencias, a identificação e abordagem dos participantes acontecerá através das redes sociais. O Facebook, Instagram e Twitter serão as bases iniciais de contato com o público-alvo da pesquisa. A técnica de coleta de dados utilizada será a de Bola de Neve.

De acordo com Flick (2009), esta técnica de coleta de dados é como a de um bom repórter que rastreia as 'pistas' de uma pessoa para outra. Inicialmente, o pesquisador especifica as características que os membros da amostra deverão ter, depois identifica uma pessoa ou um grupo de pessoas congruentes aos dados necessários, na sequência apresenta a proposta do estudo e, após obter/registrar tais dados, solicita que o(s) participante(s) da pesquisa indique(m) outra(s) pessoa(s) pertencente(s) à mesma população-alvo. Flick (2009) explica que, na técnica de Bola de Neve, o pesquisador pede ao participante referência de novos informantes que possuam as características desejadas. Esse processo continua até que 
as métricas estabelecidas antecipadamente para a coleta de dados, como prazo de coleta ou quantidade máxima de entrevistados, sejam atingidas ou ocorra saturação teórica, isto é, quando não surgem novas informações nos dados coletados.

\section{Considerações finais}

As instituições de ensino superior devem cumprir um papel importante ao não se eximirem da responsabilidade de contribuir na resposta brasileira ao enfrentamento da COVID-19. Existem várias possibilidades de atuação neste cenário de crise.

As Ligas Acadêmicas desempenham um papel importante na formação. Os estudantes possuem oportunidade de fazer escolhas de modo ativo e livre, ter iniciativas inovadoras. Ao aturem no enfrentamento da pandemia, sua práxis extensionista incorpora saberes e práticas que estão em harmonia com as necessidades impostas pelos problemas vivenciados pela sociedade.

A Universidade, ao desenvolver ações de extensão durante a pandemia, comprometese em sustentar seu valor social, mostra-se atenta para o delicado momento histórico mundial. Assim, contribuirá para a promoção do desenvolvimento social, tanto na realidade atual quanto na que se instalará no futuro pós-pandemia.

A LISAM, ao desenvolver atividades de extensão durante a pandemia, tem oportunizado aos seus integrantes um espaço fértil de aprendizagem no ensino, pesquisa e extensão, contribuindo para a formação de profissionais éticos, reflexivos e críticos, com senso de responsabilidade social e compromisso com a cidadania, capazes de perceber e acolher as diversas demandas comunitárias. Possuindo assim foco na promoção da saúde mental.

AGRADECIMENTOS: à Pró-Reitoria de Assuntos Estudantis da Universidade Estadual Vale do Acaraú (UVA) pelo apoio permanente.

\section{REFERÊNCIAS}

ARAÚJO, C. R. C. et al. Contribuição das ligas acadêmicas para a formação em enfermagem. Enfermagem em Foco, v. 10, n. 6, p. 137-142, 2019. Disponível em:

http://revista.cofen.gov.br/index.php/enfermagem/article/view/2802. Acesso em: 1 set. 2020. 
BEGO, A.; SILVA, L. A indissociabilidade entre ensino, pesquisa e extensão no PIBID. Revista Ciência em Extensão, v. 14, n. 2, p. 20-42, 2018. Disponível em:

https://ojs.unesp.br/index.php/revista_proex/article/view/1602. Acesso em: 4 set. 2020.

BRASIL. Ministério da Educação. Conselho Nacional de Educação. Câmara de Educação Superior. Resolução n⿳ 7 , de 18 de dezembro de 2018. Estabelece as Diretrizes para a Extensão na Educação Superior Brasileira e regimenta o disposto na Meta 12.7 da Lei $\mathrm{n}^{\mathrm{o}}$ 13.005/2014, que aprova o Plano Nacional de Educação - PNE 2014-2024 e daí outras providências. Brasília: Ministério da Educação, 19 dez. 2018. Disponível em: https://www.in.gov.br/materia/-/asset_publisher/Kujrw0TZC2Mb/content/id/55877808. Acesso em: 1 set. 2020.

CASTRO, M. G.; ALVES, D. A. Ensino, pesquisa e extensão na Universidade Federal de Viçosa: origem e trajetória institucional (1926-1988). Revista Brasileira de Educação, Rio de Janeiro, v. 22, n. 70, p. 752-773, jul. 2017. Disponível em:

https://www.scielo.br/pdf/rbedu/v22n70/1809-449X-rbedu-22-70-00752.pdf. Acesso em: 4 set. 2020 .

CAVAlCANTE, A. S. P. et al. As Ligas Acadêmicas na Área da Saúde: Lacunas do Conhecimento na Produção Científica Brasileira. Revista Brasileira de Educação Médica, Brasília, v. 42, n. 1, p. 199-206, jan, 2018. Disponível em: https://www.scielo.br/pdf/rbem/v42n1/0100-5502-rbem-42-01-0199.pdf. Acesso em: 9 set. 2020 .

DARSIE, C.; WEBER, D. L. Disease and space control: issues about dispersion and isolation in pandemic times? Journal of Infection Control, v. 9, n. 2, p. 47-48, abr./jun. 2020. Disponível em: http://jic-abih.com.br/index.php/jic/article/viewFile/298/pdf_1. Acesso em: 2 set. 2020 .

DUARTE, M. Q. et al. COVID-19 e os impactos na saúde mental: Uma amostra do Rio Grande do Sul. Ciência e Saúde Coletiva, Rio de Janeiro, v. 25, n. 9, p. 3401-3411, set. 2020. Disponível em: https://www.scielo.br/pdf/csc/v25n9/1413-8123-csc-25-09-3401.pdf. Acesso em: 9 set. 2020.

FIOCRUZ. Saúde mental e atenção psicossocial na pandemia covid-19: Recomendações Gerais. Rio de Janeiro: Fiocruz, 2020. Disponível em: https://www.fiocruzbrasilia.fiocruz.br/wp-content/uploads/2020/04/Sa\%C3\%BAde-Mental-eAten $\% \mathrm{C} 3 \% \mathrm{~A} 7 \% \mathrm{C} 3 \% \mathrm{~A} 3 \mathrm{o}-\mathrm{Psicossocial}-$ na-Pandemia-Covid-19recomenda\%C3\%A7\%C3\%B5es-gerais.pdf. Acesso em: 6 set. 2020.

FLICK, U. Introdução à pesquisa qualitativa. Porto Alegre: Artmed, 2009.

HUNGER, D.; ROSSI, F.; PEREIRA, J. M.; NOZAKI, J. M. O dilema extensão universitária. Educação em Revista, v. 30, n. 3, p. 335-354, jul./set. 2014. Disponível em: https://www.scielo.br/pdf/edur/v30n3/edur640.pdf. Acesso em: 25 ago. 2020.

KOLA, L. Global mental health and COVID-19. The Lancet Psychiatry, v. 7, n. 8, p. 655657, jun. 2020. Disponível em: https://www.thelancet.com/journals/lanpsy/article/PIIS22150366(20)30235-2/fulltext. Acesso em: 9 set. 2020. 
LIMA, R. C. Distanciamento e isolamento sociais pela covid-19 no Brasil: impactos na saúde mental. Physis: Revista de Saúde Coletiva, Rio de Janeiro, v. 30, n. 2, p. 1-10, 2020. Disponível em: https://scielosp.org/pdf/physis/2020.v30n2/e300214/pt. Acesso em: 4 set. 2020.

OLIVEIRA, E. N. et al. Liga interdisciplinar em saúde mental: trilhando caminhos para a promoção em saúde. Saúde em Redes, v. 5, n. 3, p. 317-327. 2019. Disponível em: http://revista.redeunida.org.br/ojs/index.php/rede-unida/article/view/2435/437. Acesso em: 3 set. 2020 .

OLIVEIRA, E. N. Saúde Mental durante a Pandemia do novo Coronavírus: algumas reflexões necessárias. Research, Society and Development, v. 9, n. 8, p. 1-18, jul. 2020. Disponível em: https://rsdjournal.org/index.php/rsd/article/view/5478. Acesso em: 4 set. 2020.

OLIVEIRA, E. N. et al. Projeto vida em quarentena: estratégia para promoção da saúde mental de enfermeiros diante da COVID-19. Enfermagem em Foco, v. 11, n. 1, p. 162-167, 2020. Disponível em:

http://revista.cofen.gov.br/index.php/enfermagem/article/view/3741/820. Acesso em: 05 set. 2020.

ORGANIZAÇÃO PAN-AMERICANA DE SAÚDE. Folha informativa - COVID-19 (doença causada pelo novo coronavírus). OPAS, Brasil, 11 de set. 2020. Disponível em: https://www.paho.org/bra/index.php?option=com_content\&view=article\&id=6101:covid19\&I temid $=875$. Acesso em: 5 maio 2020 .

PEREIRA, M. D. et al. The COVID-19 pandemic, social isolation, consequences on mental health and coping strategies: an integrative review. Research, Society and Development, v. 9, n. 7, p. 1-31. 2020. Disponível em:

https://rsdjournal.org/index.php/rsd/article/view/4548/4043. Acesso em: 9 set. 2020.

QUEIROZ, S. J. et al. A Importância das Ligas Acadêmicas na Formação Profissional e Promoção de Saúde. Revista Fragmentos de Cultura, Goiânia, v. 24, n. esp., p. 73-78, nov. 2014. Disponível em: http://seer.pucgoias.edu.br/index.php/fragmentos/article/view/3635. Acesso em: 9 set. 2020.

RIBEIRO, J. L. P. Inventário de saúde mental. Lisboa: Placebo Editora, 2011.

SANTOS, D. R.; VIEIRA, L. C. Importância da interdisciplinaridade no ensino superior. Revista Faculdade Montes Belos, v. 4, n. 2, p. 1-13, nov. 2011. Disponível em: http://revista.fmb.edu.br/index.php/fmb/article/view/41. Acesso em: 5 set. 2020.

SCHMIDT, B. et al. M. Saúde mental e intervenções psicológicas diante da pandemia do novo coronavírus (COVID-19). Estudos de psicologia, Campinas, v. 37, p. 1-13, 2020. Disponível em: https://www.scielo.br/pdf/estpsi/v37/1678-9865-estpsi-37-e200063.pdf. Acesso em: 6 set. 2020.

SERRÃO, A. C. P. Em Tempos de Exceção como Fazer Extensão? Reflexões sobre a Prática da Extensão Universitária no Combate à COVID-19. Revista Práticas em Extensão, São Luís, v. 04, n. 1, p. 47-49, jun. 2020. Disponível em: 
http://ppg.revistas.uema.br/index.php/praticasemextesao/article/view/2223. Acesso em: 3 set. 2020.

SHIOZAWA, P.; UCHIDA, R. R. Social media during a pandemic: bridge or burden? São Paulo Medical Journal, São Paulo, v. 138, n. 3, p. 267-268, jun. 2020. Disponível em: http://www.scielo.br/scielo.php?script=sci_arttext\&pid=S1516$31802020000300267 \& 1 n g=e n \& n r m=$ iso. Acesso em: 9 set. 2020.

SILVA, A. R. Oportunidades para Extensão Universitária nos Tempos de Pandemia COVID-19. Revista práticas em extensão, São Luís, v. 4, n. 1, p. 40-41, jun. 2020. Disponível em: http://ppg.revistas.uema.br/index.php/praticasemextesao/article/view/2181. Acesso em: 3 set. 2020.

SILVA, D. A. et al. Educação em enfermagem: criação de uma liga acadêmica para o ensino de urgência e emergência. Research, Society and Development, v. 9, n. 3, p. 1-11, mar. 2020. Disponível em: https://rsdjournal.org/index.php/rsd/article/view/2656. Acesso em: 9 set. 2020 .

SILVA, R. M. G.; CAMPANI, A.; NEGREIROS, J. G. Contribution of extension to an innovative university teaching: a study from the nursing league program of Vale do Acaraú State University. Revista Ibero-Americana de Estudos em Educação, Araraquara, v. 15, n. esp., p. 1615-1628, ago. 2020. Disponível em:

https://periodicos.fclar.unesp.br/iberoamericana/article/view/13835. Acesso em: 3 set. 2020.

SILVA, S. A.; FLORES, O. Ligas Acadêmicas no Processo de Formação dos Estudantes. Revista Brasileira de Educação Médica, Rio de Janeiro, v. 39, n. 3, p. 410- 417, jul./set. 2015. DOI: https://dx.doi.org/10.1590/1981-52712015v39n3e02592013

SOUADKA, A. et al. COVID-19 and Healthcare worker's families: behind the scenes of frontline response. EClinicalMedicine, v. 23, p. 100373, maio 2020. Disponível em: https://www.thelancet.com/journals/eclinm/article/PIIS2589-5370(20)30117-6/fulltext. Acesso em: 04 set. 2020.

STATISTA. Twitter - Statistics \& Facts. Disponível em: https://www.statista.com/topics/737/twitter/. Acesso em: 18 ago. 2020

\section{Como referenciar este artigo}

OLIVEIRA. E. N.; ANDRADE, C. S. G.; LIMA, L. M. C. A liga interdisciplinar em saúde mental e suas contribuições em tempos de covid-19. Doxa: Rev. Bras. Psico. e Educ., Araraquara, v. $22, \quad$ n. $2, \quad$ p. 410-423, jul./dez. 2020. e-ISSN: 2594-8385. DOI: https://doi.org/10.30715/doxa.v22i2.14171

Submetido em: $30 / 04 / 2020$

Revisões requeridas: $20 / 06 / 2020$

Aprovado em: $14 / 07 / 2020$

Publicado em: 31/08/2020 